\title{
Scanning Electrochemical Microscopy
}

\author{
Cyrine SLIM, Sophie GRIVEAU and Fethi BEDIOUI
}

Chimie ParisTech, PSL Université Paris, Unité de Technologies Chimiques et Biologiques

pour la Santé, CNRS 8258, INSERM 1022, Université Paris Descartes, 75005 Paris, France

\begin{abstract}
Scanning electrochemical microscopy (SECM) is a method of local probe microscopy based on the displacement of an ultramicroelectrode (UME) in the vicinity of an interface. The UME has at least one dimension in the micrometer range. It is extremely useful in modern electroanalysis for the characterization of surfaces by imaging electroactive and nonelectroactive materials or for obtaining quantitative data on specific analytes or processes by studying the reactions occurring on the surface of a substrate. It operates through several modes (feedback, generation/collection, penetration etc...) and allows evaluating kinetics of chemical reactions, studying biological cells, achieving localized surface modifications or imaging surfaces. More recently, scanning electrochemical cell microscopy (SECCM) was developed to allow obtaining high spatial resolution images. SECCM is derived from SECM and consists of a probe which is a sharp double barrel capillary (nanopipette), with both compartments containing a quasi-reference electrode or quasi-reference counter electrode (QRCE) and filled with an electrolytic solution.
\end{abstract}

Key words: ultramicroelectrodes, amperometry, approach curves, scanning, imaging, feedback mode, generation/collection 


\section{Introduction}

Scanning electrochemical microscopy (SECM) is a method of local probe microscopy ${ }^{1,2}$ based on the displacement of an ultramicroelectrode (UME) in the vicinity of an interface. The UME has at least one dimension in the micrometer range in order to overcome the limitations of conventional electrodes in respect of charging current and uncompensated resistance and it may initiate the electrochemical reaction of interest.

The instrumentation, theory, modes and initial applications of SECM were developed in Bard's laboratory during the 1980's. It has rapidly become more than just an electrochemical method but rather a different way of doing electrochemistry ${ }^{3}$. In fact, almost any kind of electrochemical measurement, like cyclic voltammetry, ac voltammetry or potentiometry, may be carried out in SECM. The spatial resolution of this technique greatly increases the utility of electrochemistry for the characterization of interfaces.

SECM is extremely useful in modern electroanalysis for the characterization of surfaces by imaging electroactive and non-electroactive materials ${ }^{4,5}$, or for obtaining quantitative data on specific analytes or processes by studying the reactions occurring on the surface of a substrate. $^{6}$ It also allows the local modification of samples via electrodissolution ${ }^{7}$ or electrodeposition. ${ }^{8,9}$ Thus, advances in the miniaturization and in the diversification of UMEs offer an increasingly wide range of applications in diverse fields such as materials, ${ }^{10}$ biology, ${ }^{11,12}$ or corrosion. ${ }^{13}$

\section{Principle and Instrumentation of SECM}

SECM involves measurement of the current through the UME (also called tip or probe, with a radius, $a$, of the order of a few nanometers to $25 \mu \mathrm{m}$ ) when it is held or moved in a solution in the vicinity of a substrate. Substrates range from solid surfaces, including glass, metal, polymer, and biological material, to liquids such as mercury and immiscible oil. The presence of the substrate at a short distance from the UME perturbs the electrochemical response of the tip, which provides information about the nature and properties of the substrate itself, with a resolution depending on the UME size and with a sensitivity dictated by the minimum current that can be measured accurately.

Figure 1 illustrates an SECM instrument. It is mainly composed of an electrolytic cell, probe, sample, bipotentiostat, piezoelectric controllers, and computer. The piezoelectric controllers are employed to regulate the position of probe and substrate. The bipotentiostat allows the simultaneous control of the probe and the substrate potentials. The UME is immobilized on a holder controlled by the three-dimensional controllers, which moved along the $\mathrm{X}, \mathrm{Y}, \mathrm{Z}$, directions. For specialized applications involving a biological sample, for example, additional components (e.g., an inverted microscope, a distance control system) may be added. The tip/substrate assembly is immersed in an electrolytic solution generally containing an electroactive species (which can be dioxygen dissolved in solution) and the substrate is placed at the bottom of the electrochemical cell. A reference electrode and/or a counter-electrode are also used to control the potential applied to the UME probe and to measure the current, respectively. The bipotentiostat performs these measurements, by controlling the potential of the UME and, if necessary, that of the substrate. The detected signal corresponds to the current measured at the UME while it is scanned over the substrate. The measured current as well as its variations during the displacement of the UME above the substrate will depend on several parameters: the UME/substrate distance, the surface conductivity of the substrate, its electrochemical reactivity and its roughness.

The most common mode of operation of the SECM is to collect the electrochemical signal at the UME that will depend on the distance between the UME and the nature of the surface to 
be analyzed. An understanding of the operation of the SECM and of its quantitative aspects depends upon an understanding of electrochemistry at UMEs. UMEs with disk geometry are most important for SECM and the discussion will be restricted to this geometry. A discshaped UME consists of a conductive wire sealed in a glass capillary (platinum wire, gold or carbon fiber) (Figure 2A). When the UME is introduced in an electrolytic solution containing an electroactive species, for example a reductant $(\mathrm{R})$ and held immobile far from any surface, the shape of the typical voltammogram (Figure 2B) is that of a sigmoid with the appearance of a steady-state current $i_{\mathrm{T}, \infty}$ given by the equation:

$$
i_{T, \infty}=4 n F D_{\text {Red }} C_{\text {Red }}^{\text {sol }} a
$$

where $n$ is the number of electrons, $F$ is the Faraday constant $\left(96500 \mathrm{C} \mathrm{mol}^{-1}\right), D_{\text {Red }}$ is the diffusion coefficient of the reductant $\left(\mathrm{cm}^{2} \cdot \mathrm{s}^{-1}\right)$ in the bulk solution, and $C_{\text {red }}^{\text {sol }}$ the reductant bulk concentration $\left(\mathrm{mol} . \mathrm{cm}^{-3}\right)$. Equation 1 is valid at constant potential, sufficiently positive to induce the immediate oxidation of $\mathrm{R}$ in $\mathrm{O}$ to the electrode $\left(\mathrm{E}-\mathrm{E}^{\circ}>60 \mathrm{mV}\right)$. When the UME slowly approaches near a sample, the presence of the latter will disturb the diffusion of the species $\mathrm{R}$ at the tip and consequently the measured current so-called $i_{\mathrm{T}}$.

\section{SECM modes}

\subsection{The feedback mode}

The most frequent mode of operation of the SECM is the feedback mode, where only the tip current is monitored. Here, the tip current is perturbed by the presence of a substrate at close proximity by blockage of the diffusion of solution species to the tip (negative feedback) and by its regeneration at the substrate (positive feedback). This effect permits investigation of both electrically insulating and conducting surfaces and makes possible imaging of surfaces and the reactions that occur there. ${ }^{14,15}$ In the feedback mode, as a tip approaches a comparatively large substrate in the $\mathrm{z}$ direction, the steady-state tip current $\left(i_{\mathrm{T}}\right)$ depends on the tip-substrate separation distance $(d)$ and on whether the substrate is an insulator or conductor. When the tip is very far from the substrate (e.g., $d$ is greater than 10 times the tip radius $(a)$ ), the measured tip current $\left(i_{\mathrm{T}, \infty}\right)$ for the reaction $\mathrm{O}+\mathrm{ne} \rightarrow \mathrm{R}$ is diffusion controlled (Fig. 3A) and $i_{\mathrm{T}, \infty}$ is constant and given by equation 1 .

For $d<10 a$, the presence of the underlying substrate affects $i_{\mathrm{T}}$ significantly. For an insulating substrate, the concentration of $\mathrm{O}$ in the tip-substrate gap decreases relative to that in the bulk solution since diffusion of $\mathrm{O}$ into the gap from the bulk solution is hindered. Thus, $i_{\mathrm{T}}$ decreases as the tip moves closer to the insulating substrate (i.e., $d$ decreases) in this negative feedback mode (Fig. 3B). In contrast, as the tip approaches a conducting substrate, tipgenerated $\mathrm{R}$ is re-oxidized back to $\mathrm{O}$ at the substrate with an increase in the local flux of $\mathrm{O}$ as $d$ decreases, and a corresponding increase in $i_{\mathrm{T}}$ in this positive feedback mode (Fig. 3C). A plot of normalized current $\left(i_{\mathrm{T}}=i_{\mathrm{T}} / i_{\mathrm{T}, \infty}\right) v s$. normalized distance $(L=d / a)$ results in approach curves for either positive (black) and negative feedback (red) (Fig. 3D). Analytical expressions of positive and negative feedback curves have been proposed by several groups, allowing to accurately locate the tip with respect to the sample. ${ }^{16,17}$ Indeed, from comparison of experimental and simulated curves, $d$ can be established from the measured $i_{\mathrm{T}}$ and also the value of $d=0$. The approach curve for an insulator sample depends on the tip RG (i.e., the ratio of the tip insulating sheath radius $r_{\mathrm{g}}$ to its conducting radius $a$ or $r_{\mathrm{g}} / a$ ) since the sheath around the conducting portion of the tip also blocks diffusion, but this effect is not usually important for positive feedback. ${ }^{18,19}$

\subsection{The Generation/collection modes}

Generation/collection G/C modes involve a four-electrode system, using the tip and the substrate as working electrodes. Two types of $\mathrm{G} / \mathrm{C}$ modes are notable: the tip 
generation/substrate collection (TG/SC) mode and the substrate generation/tip collection (SG/TC) mode. ${ }^{20}$ In the TG/SC mode, the tip is used to generate a reactant that is detected at the substrate electrode (Fig. 4A). For example, the tip is held at a potential where the reaction $\mathrm{O}+$ ne $\rightarrow \mathrm{R}$ occurs. The substrate is held at a different potential where the product Red will react and thus be collected. In most cases, the substrate is considerably larger than the tip, so that the collection efficiency, given by the ratio $\mathrm{i}_{\mathrm{S}} / \mathrm{i}_{\mathrm{T}}$, is essentially 1 (corresponding to $100 \%$ of collection) for a stable tip-generated species, Red, and if the tip is close enough to the substrate (i.e, on the order of two tip radii).

The alternative mode is the substrate generation/tip collection mode (SG/TC) (Fig. 4B). In this mode, the tip probes the reactions that are occurring on a substrate. For example, the collection of the tip current while it is scanned in the z-direction can monitor the concentration profile, while a scan over the surface can identify "hot spots" where reactions occur at high rates. The SG/TC mode was first used to study concentration profiles near an electrode surface in a specific location without scanning or imaging. ${ }^{21,22,23}$. This can be used to for the screening of electrocatalysts. Usually in the SG/TC mode, the collection efficiency by the tip is smaller than 1 , so the system has to be calibrated with a known mediator couple.

\subsection{The penetration mode}

The penetration mode involves the introduction of the UME into a soft material microstructure, such as a thin film of polymer containing a redox mediator, biological cells or liposomes. The probe will allow collecting information on the variations of concentrations and the parameters of mass transport inside the material, as a function of the depth of penetration of the UME. The UME can penetrate axially in the $\mathrm{Z}$ direction into soft films, gels or biological issues; however, in these structures it can not be moved laterally. X-Y imaging is not possible without damaging the target or breaking the tip. A one-dimensional penetration experiment can serve to collect information about concentration profiles or to investigate the

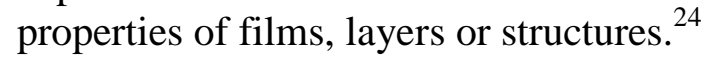

\section{Main applications of the SECM \\ 4.1. Surface imaging}

As a scanning probe technique, SECM is uniquely able to provide both chemical and topographic information of a surface immersed in an electrolytic solution. It is possible to obtain a three-dimensional image of a sample surface by scanning the tip in the X-Y plane and monitoring the measured tip current, $i_{\mathrm{T}}$, as a function of tip location, in the so-called constant-height mode. The image can be converted to a plot of Z-height (distance between tip and substrate, $d$ ), versus X-Y position via an $i_{\mathrm{T}}$ versus $d$ calibration plot. It can also be plotted as a gray-scale image, where high values of $i_{\mathrm{T}}$ are shown in light colors and small values as dark colors.

Figure 5 illustrates the correlation between surface topography and current measured at the probe and illustrates the UME path for constant height SECM image.Specifically, the probe sweeps the surface line by line at a relatively low speed rate to ensure that the tip measurements are stable (speed rate in the range between 5 and $10 \mu \mathrm{m} . \mathrm{s}^{-1}$ ). In general, measurements made at UME are relatively insensitive to agitation of the solution or other convective effects. However, the scanning of immobile sample at too high speeds causes a shear of the solution between the UME and the solution, which adds a convective component to the material transfer in addition to the diffusive component. The layer in which the material transfer is carried out is then "flattened" at the UME which results in reducing the interaction between the UME and the substrate, and hence a lower sensitivity of measurement. At too high a speed ( 2 to $6 \mathrm{~mm} \mathrm{~s}^{-1}$ ), it is no longer possible to distinguish areas of the surface with electrochemical (insulating or conductive) activity. It is therefore better to image at low 
speeds. Also the realization of surface imaging by SECM can be relatively long compared to other imaging techniques.

\subsection{Determination of kinetics of chemical reactions}

Kinetics of reactions can be studied in the TG/SC mode. Indeed, as mentioned above, for stable species generated by the UME by a simple charge transfer process, the collection efficiency (defined by the $i_{\mathrm{S}} / i_{\mathrm{T}}$ ratio) is close to $1(100 \%)$ when the distance of the substrate/UME is sufficiently small, typically at a distance $d \leq 2 a$. Thus, in the case of a chemical reaction coupled with charge transfer, for example, where the species produced at the probe reacts in the space between the probe and the substrate by generating an electroinactive species, the $i_{\mathrm{S}} / i_{\mathrm{T}}$ ratio decreases, and this variation provides access to the kinetics of the coupled chemical reaction. Depending on the speed of the coupled chemical reaction, the ratio $i_{\mathrm{S}} / i_{\mathrm{T}}$ will tend to 1 (slow chemical reaction), or to 0 in the case of a very fast chemical reaction.

\subsection{Studies of biological systems}

One of the major advantages of the SECM compared to other near-field microscopies is the ability to image directly, at the micrometer scale (and nanometrically depending on the size of the UME used) and non-invasively (no contact between the tip and the sample), living organisms (cells) under in vivo conditions, without any specific sample preparation. SECM provides access to quantitative data, for example the concentrations of electroactive species (or not) near a biological system when subjected to physical or chemical stimuli. Thus, soon after its invention, biological systems and more precisely the processes in which they are involved have been studied by SECM. ${ }^{25,26}$ This attractiveness for the SECM method is related to its non-destructive character and its ability to provide complementary information to other analysis techniques such as fluorescence imaging.

For example, the respiratory and photosynthesis activities of plants were studied by SECM in $\mathrm{SG} / \mathrm{TC}$ mode, by following the dioxygen reduction current at the probe close to the cells. ${ }^{27}$ Thanks to these measurements, it was possible to map $\mathrm{O}_{2}$ fluxes associated with cell respiration and photosynthesis. These experiments are therefore conducted with and without light irradiation, in order to show the photosynthetic activity. Using a micrometric probe (tip of radius $\sim 1.2 \mu \mathrm{m}$ ), the activity of an individual cell (protoplast) was analyzed. The SECM thus offers the possibility of studying cell metabolism at the level of the single cell.

According to a similar principle, the susceptibility of different tumor cell lines to anticancer agents was analyzed, based on analysis of cell activity by the dioxygen reduction current measurements, as illustrated on Figure $6 .^{28}$ This simple strategy is quite interesting and complementary to usual biological techniques (such as fluorescence). It can be used in for the screening of anticancer drugs. These SECM studies, offering the possibility of non-invasive, real-time cell viability measurements, have also been extended to the study of bovine embryo viability. $^{29}$

\subsection{Localized surface modification}

The SECM can be used for the localized modification of surfaces and for the realization of microscopic deposits located on both conductive and semiconducting surfaces. In this case, the UME participates directly to this modification. ${ }^{30,31}$ A variety of microstructures can be envisioned, ranging from the microscopic electrodeposition of metals and polymers to electrochemical dissolution of metals and the modification of chemical functions on the surface. Two main categories based on the mode of operation are illustrated in Figure 7 . In the direct mode, the substrate serves as the auxiliary electrode, while in the feedback mode the substrate is unbiased (in most cases). In both modes, the lateral displacement of the probe during the surface modification allows the creation of patterns on the surface of the substrate. The direct mode principle (Figure 7A) is restricted to conductive samples. It was used for example for localized metal deposition and etching ${ }^{32-36}$ or the localized polymer deposition such as 
polypyrrole and polyaniline. ${ }^{37,38}$ The feedback mode (Figure 7B) was used for example to modify the surface of fluorinated polymers, and in particular Teflon ${ }^{\circledR}$ or Dyneon ${ }^{\mathrm{TM}}$ by inducing their local reduction. ${ }^{39,40}$ The main advantage of the feedback mode over the direct mode lies in the possibility of modifying any type of surface (conductive or semiconducting).

\section{Improvement of the resolution of the electrochemical imaging: case of scanning electrochemical cell microscopy}

Scanning electrochemical cell microscopy (SECCM) is a scanning-droplet-based method conceived for the high-resolution electrochemical imaging of surfaces. This technology is derived from SECM and scanning ion conductance microscopy and was recently developed by the group of Unwin. ${ }^{41,42,43,44}$ Its principle is based on the use of a mobile nano electrochemical cell allowing high spatial resolution images to be obtained.

The SECCM instrumentation is illustrated in Figure 8A and operates under the constantdistance mode between the nanosized SECCM probe and the sample, to avoid contact between the probe and the sample. The SECCM probe is a sharp double barrel capillary (nanopipette), with both compartments containing a quasi-reference electrode or quasireference counter electrode (QRCE) and filled with an electrolytic solution (Figure 8B ${ }^{45}$ ). By applying a low potential between the two QRCE, an ion migration current is generated across the thin liquid meniscus at the orifice of the probe. Simultaneously, a small amplitude oscillation of the probe is added perpendicularly to the sample surface (Figure 8B). As for SECM, the first step consists of approaching the tip of the sample surface. In initial stage, the capillary is positioned far from the substrate (in air) and the hanging liquid that formed at the end of the pipette is not in contact with the sample surface. When the liquid meniscus enters in contact with the sample surface, an alternating conductance current is created between the barrels with the same frequency of oscillation due to the movement of the thin liquid layer between the probe and the sample. Any change in the alternating current is indicative of a modification of the distance between the probe and the sample surface. This alternating current is therefore used as the feedback signal to keep constant the distance between the probe and the sample during the displacement of the probe above the sample, to realize imagery at constant distance and avoid any crash of the probe on the sample surface. It should be noted that the sample is not immersed in solution during SECCM experiment since the electrochemical cell is confined and defined between the protruding liquid at the probe end and the sample and nanometric to macroscopic, (semi)conducting or insulating, soft and solid samples can be interrogated. The lateral movement of the tip above the sample surface drives that of the nano-electrochemical cell, thus limiting potential fouling or passivation of the sample surface during experiments.

The re-positioning of the probe during the sample mapping is used for the analysis of the apparent topographical features of the sample, from the values of the vertical displacement of the probe during scanning. The probe is maintained in close vicinity to the sample surface but without contact (typically $25 \%$ of the probe radius). The monitoring of migration current during scanning can be used to map the local conductance of the samples. In the case of conducting and semi-conducting samples, constituting the main materials studied by SECCM, the electrochemical activity can also be simultaneously evaluated during the moving of the probe above the sample, by application of a potential between the sample and one of the QRCEs.

\subsection{SECCM probe}

The SECCM probe is a double-barrel pipette with a sharp end opening with a micro- to nanometric opening, obtained by pulling the tetha capillary (quartz or borosilicate) with a laser puller. Each barrel is filled with an electrolytic solution, typically an aqueous solution with a 
ionic strength of 10 to $50 \mathrm{mM}$ and contains a QRCE, typically $\mathrm{Ag} / \mathrm{AgCl}$ electrode (Figure 8B).

The spatial resolution of the SECCM is directly related to the diameter of the pulled doublebarrel capillary orifice. Indeed, the size of the working electrode, defined by the contact zone between the sample and the hanging liquid at the tip of the capillary, is directly related to the diameter of the probe. This diameter currently lies between $100 \mathrm{~nm}$ and $10 \mu \mathrm{m}$. An example of a nanopipette is illustrated in the electron microscopy image of Figure $8 C^{46}$. For the evaluation of the electrochemical activity of conducting and semi-conducting surfaces, a redox probe (typically ferrocene, ferrocene derivatives, hexaamineruthenium(III) choride etc) is often added into the electrolytic solution. A potential is then applied between the sample material (acting as the working electrode) and one of the QRCE (acting as the reference electrode) to drive the desired electrochemical reaction.

\subsection{Instrumentation and Imagery}

Similarly to SECM, several components are needed for the full instrumental setup (Figure 8A). High-resolution piezoelectric positioners are used for the accurate positioning of the probe. Two positioning configurations are possible: $(i)$ a vertical $(\mathrm{Z})$ positioning of the nanopipette and a $2 \mathrm{D}(\mathrm{X}, \mathrm{Y})$ positioning of the sample or $(i i)$ the $3 \mathrm{D}(\mathrm{x}, \mathrm{y}, \mathrm{z})$ positioning of the nanopipette (the sample being stationary). The first configuration is generally preferred to avoid cross talk between piezoelectric positioners. A constant separation between the probe and the sample should be kept during imaging to prevent the crash of the sharp probe. The $\mathrm{Z}$ positioning is used for the sinusoidal modulation of the tip, with an oscillation amplitude of a small fraction of the probe diameter, typically $\sim 50-100 \mathrm{~nm}$. During the mapping of the sample properties, the distance between the sample surface and the probe is maintained at about $25 \%$ to $50 \%$ of the probe radius. The SECCM instrument should be isolated from vibration, acoustic and electric noise, so that the instrument should be installed on antivibration table within a Faraday cage equipped with acoustic foam. SECCM setups are predominantly home-made apparatus (Figure 8A).

\subsection{Main applications}

Most of the reported SECCM studies have been dedicated to the analysis of electrochemical activity and electrocatalytical properties of conducting and semi-conducting (nano)materials. For example, SECCM was applied to the mapping with high-resolution of the electron transfer kinetics of simple electrochemical systems at individual pristine single-walled carbon nanotubes (SWCNTs). To this end, a narrowed SECCM probe was used, with a diameter of $400 \mathrm{~nm}$ (Figure 9A) ${ }^{47}$. It was also used as an innovative tool for the nano and microscale localized surface functionalization and modification of solid samples. For example, polyaniline motifs were locally deposited on gold surface by SECCM, as dots or nanometric bands ( $400 \mathrm{~nm}$ wide), as illustrated on Figure $9 \mathrm{~B}^{48}$. SECCM was also applied to the local electrografing of aryldiazonium salt on an HOPG surface, allowing the deposition of an array of reproducible spots of aryl layers of $\sim 1 \mu \mathrm{m}$ diameter obtained with a pipette with a $1 \mu \mathrm{m}$ orifice (Figure 9C) ${ }^{49}$. 


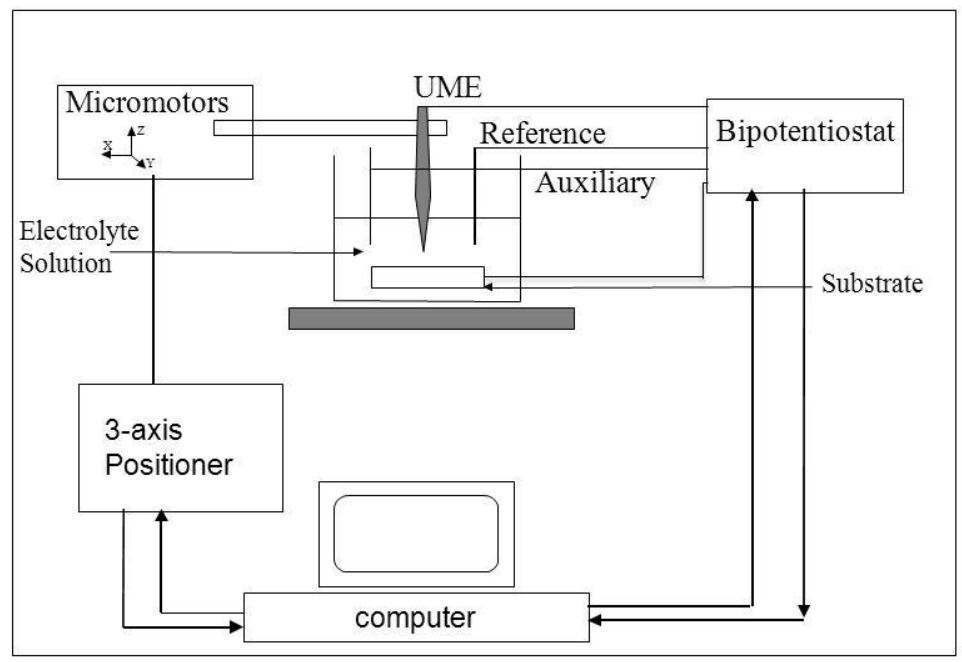

Figure 1 An illustration of an SECM instrument. 


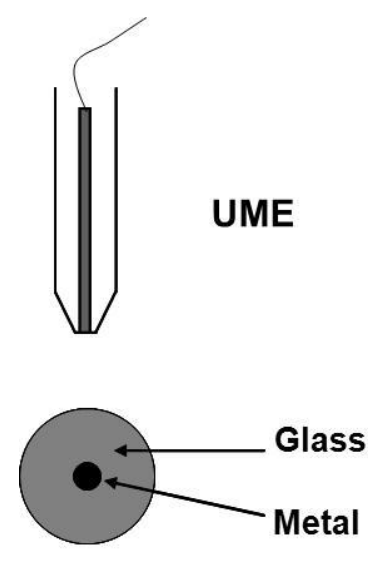

(A)

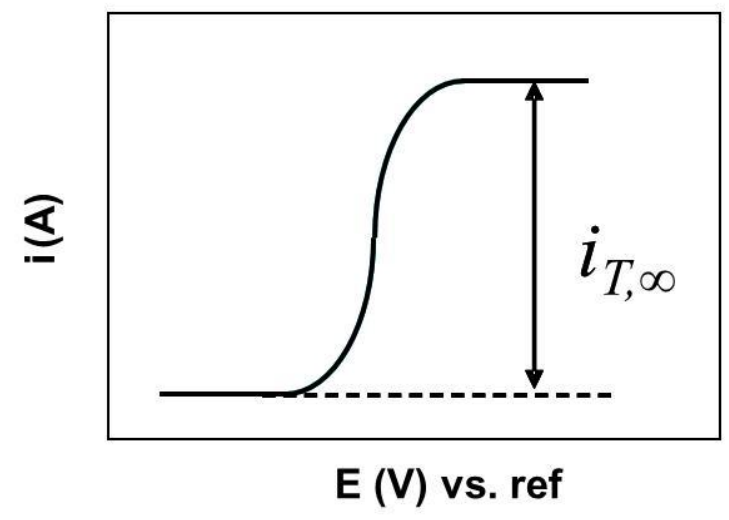

(B)

Figure 2: (A) Ultramicroelectrode with disc-shaped geometry; (B) Typical voltammogram for an ultramicroelectrode 


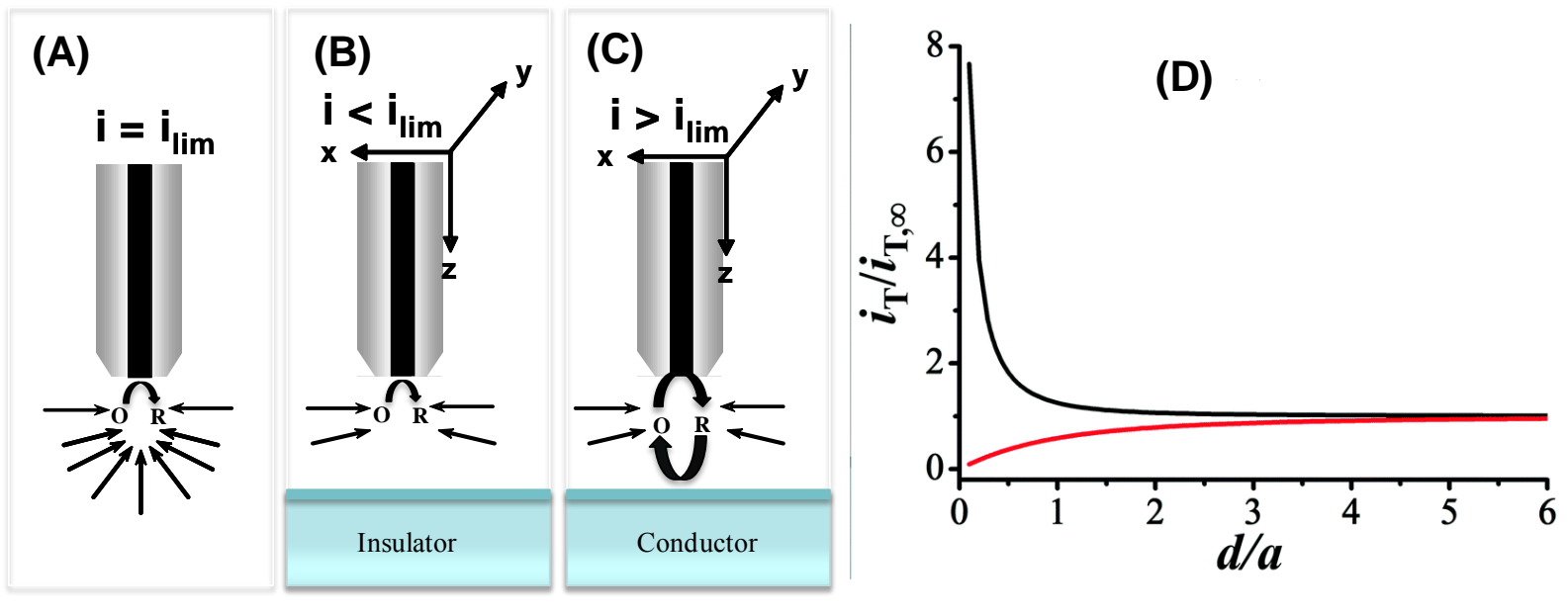

Figure 3 SECM feedback modes. (A) Unhindered diffusion of species $\mathrm{O}$ in the bulk solution to a UME tip surface when the tip is far from a substrate. (B) Hindered diffusion of species $\mathrm{O}$ to the tip by an insulating substrate (negative feedback). (C) Increased local flux of $\mathrm{O}$ by a conducting substrate (positive feedback). (D) Representative negative feedback (red) and positive feedback (black) approach curves. 

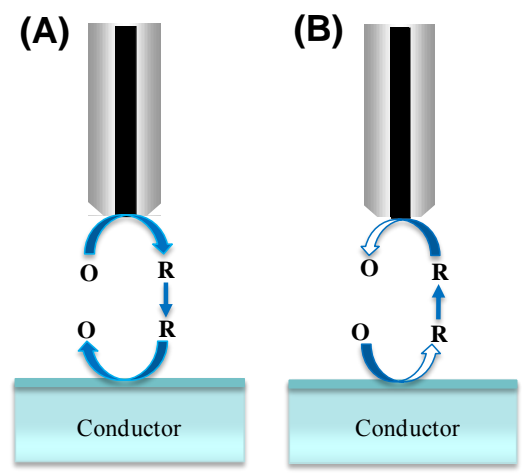

Figure 4 SECM generation/collection (G/C) modes. (A) Tip generation/substrate collection (TG/SC) and (B) substrate generation/tip collection (SG/TC). 


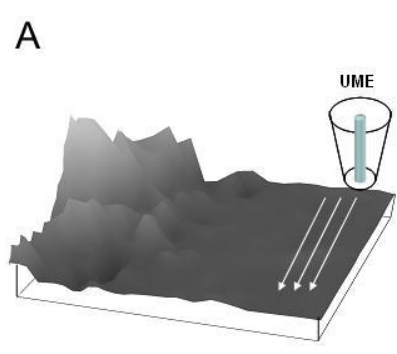

(A)
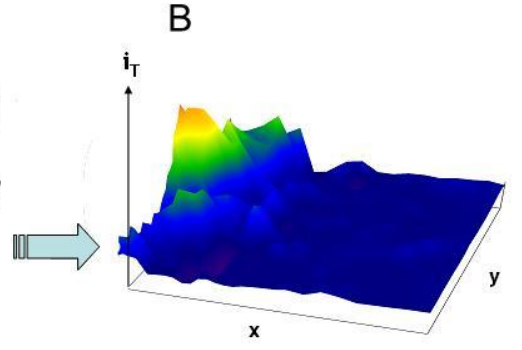

(B)

Figure 5. (A) A constant-height 3D SECM image of a carbon surface (B) the corresponding response of $i_{\mathrm{T}, \infty}$ in feedback mode 
(A)

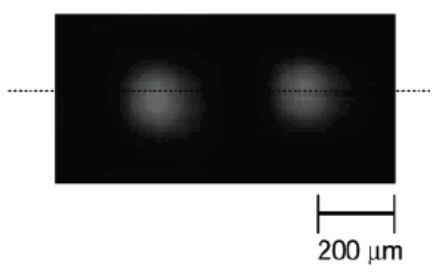

(B)

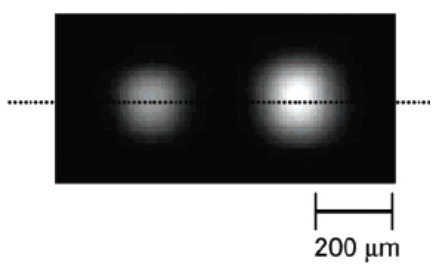

80

Decrease of $\mathrm{O}_{2}$ reduction current $(\mathrm{pA})$

Figure 6. SECM imaging of cancer cells before (A) and after (B) three days of treatment with an anticancer agent. The very clear stain is indicative of a strain of cells resistant to anticancer molecules, because it reflects a low current of oxygen reduction available near these cells (and therefore a strong respiratory activity). Adapted with permissions from reference 30. 


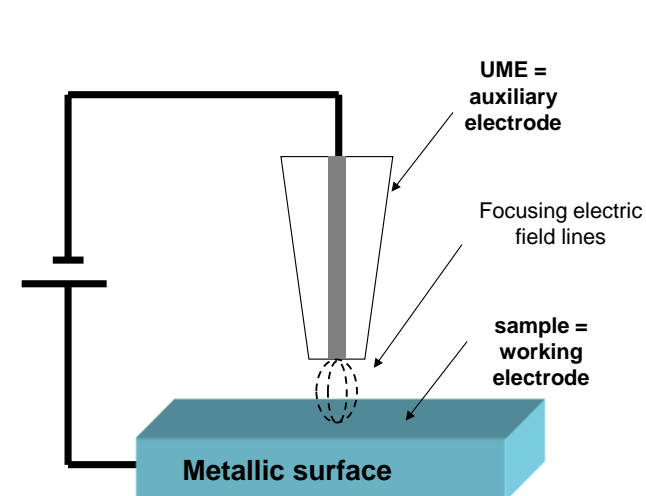

(A)

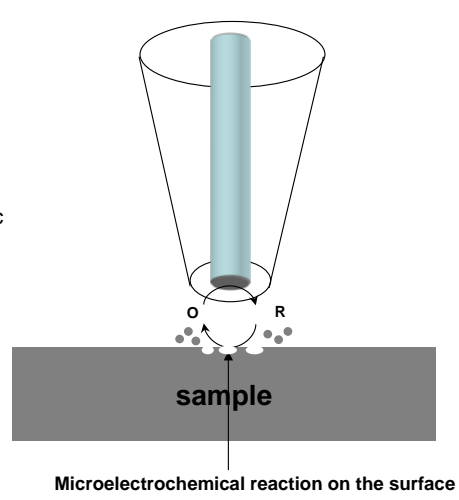

(B)

Figure 7. Schematic representation of the direct mode (A) and the feedback mode (B) of the SECM for the localized modification of the substrate surface. 

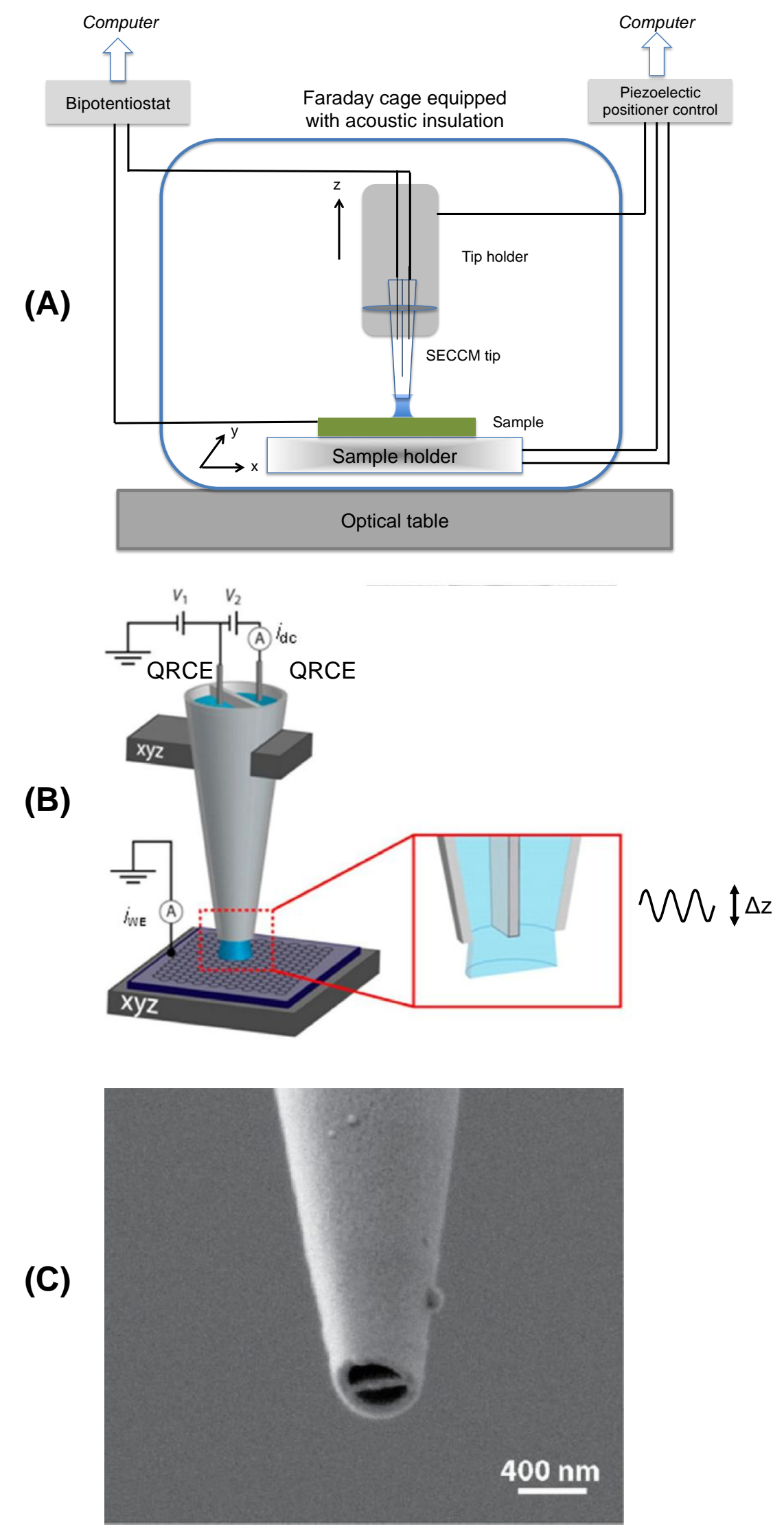

Figure 8. (a) SECCM instrument (b) SECCM principle using a dual barrel nanopipette as probe Adapted with permission from reference 47 (c) Electronic microscopy image of a dual barrel SECCM nanopipette. Adapted with permission from reference 48 

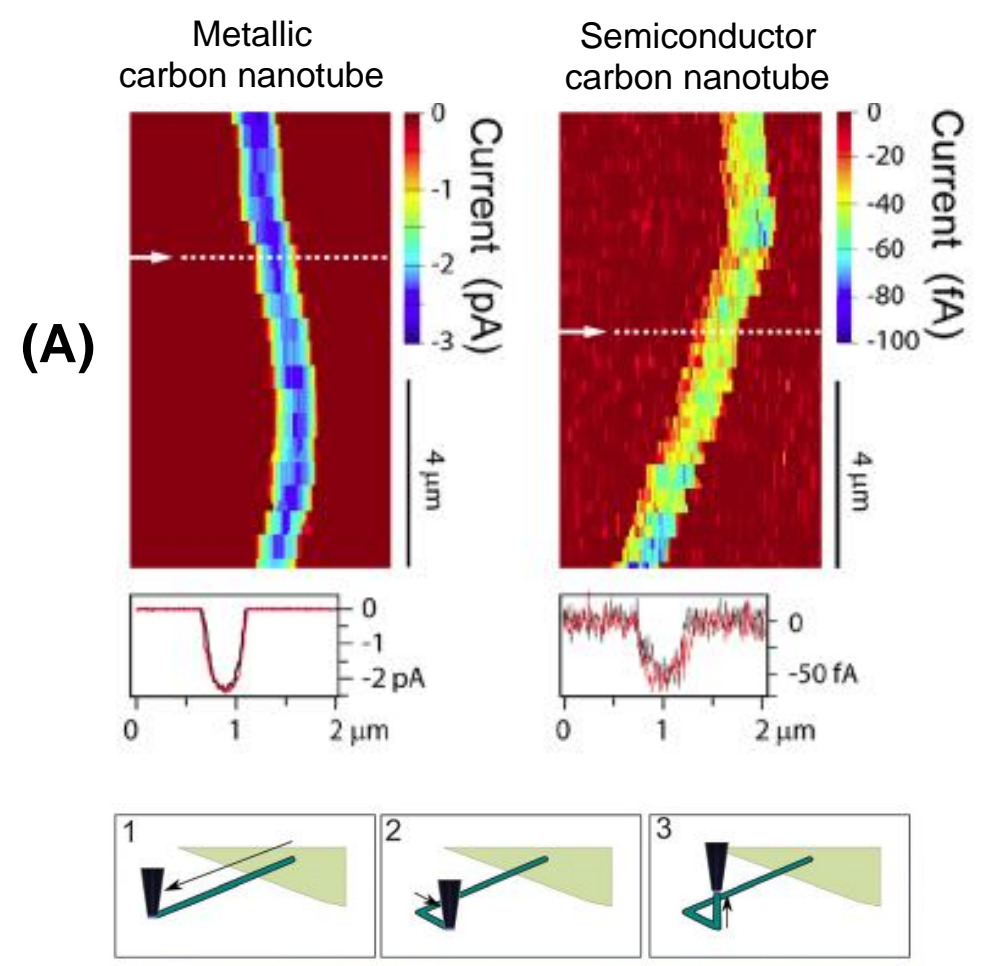

(B)
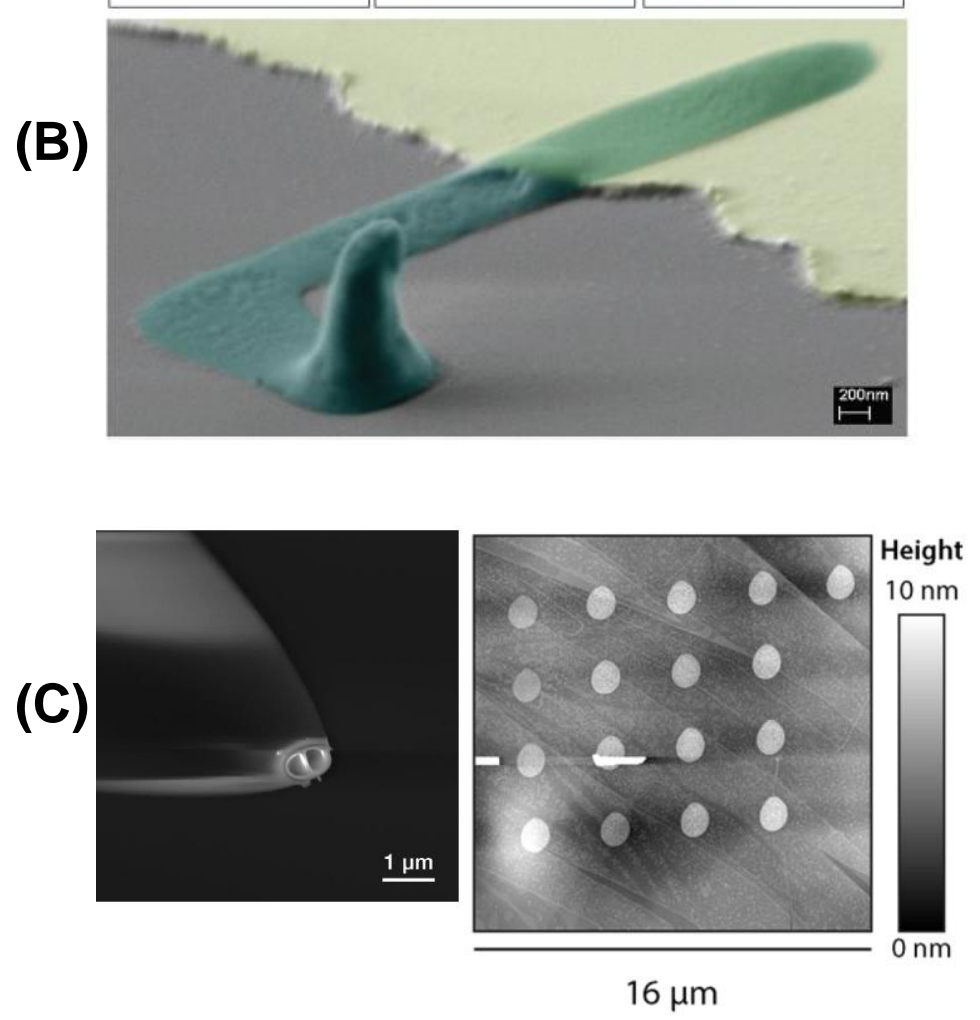

Figure 9. (a) Local electrochemical activity assessed by SECCM of conducting and semiconducting SWCNT (using $\mathrm{Ru}\left(\mathrm{NH}_{3}\right)_{6}{ }^{3+}$ as the redox mediator). Adapted with permission from reference 49 (b) Scanning electronic microscopy image of a localized deposit of polyaniline on conductive and insulating domains of a sample using SECCM Adapted with permission from reference 50 (c) Left : pipette used for diazonium electrografting; Right : AFM imaging of an array of sub-micrometric sized aryl layers deposited thanks to SECCM from aryldiazonium electrografting. Adapted with permission from reference 51 


\section{References}

\footnotetext{
${ }^{1}$ Lillehei PT, Bottomley LA (2000) Scanning probe microscopy. Analytical Chemistry 72: 189-196.

${ }^{2}$ Poggi MA, Bottomley LA, and Lillehei PT (2002) Scanning probe microscopy. Analytical Chemistry 74: 2851-2862.
}

${ }^{3}$ Mirkin MV, Horrocks BR (2000) Electroanalytical measurements using the scanning electrochemical microscope. Analytica Chimica Acta 406: 119-146.

${ }^{4}$ Yamashita K, Takagi M, Uchida K, Kondo H, and Takenaka S (2001) Visualization of DNA microarrays by scanning electrochemical microscopy (SECM). Analyst 126: 1210-1211.

${ }^{5}$ Kranz C, Wittstock G, Wohlschläger H, and Schuhmann W (1997) Imaging of microstructured biochemically active surfaces by means of scanning electrochemical microscopy. Electrochimica acta 42: 3105-3111.

${ }^{6}$ Kranz C, Lötzbeyer T, Schmidt H-L, and Schuhmann W (1997) Lateral visualization of direct electron-transfer between microperoxidase and electrodes by means of scanning electrochemical microscopy. Biosensors \& Bioelectronics 12: 257-266.

${ }^{7}$ Macpherson JV, and Unwin PR (1996) Scanning Electrochemical Microscope-Induced Dissolution: Theory and Experiment for Silver Chloride Dissolution Kinetics in Aqueous Solution without Supporting Electrolyte. Journal of Physical Chemistry 100: 19475-19483.

${ }^{8}$ Marck C, Borgwarth K, and Heinze J (2001) Generation of Polythiophene Micropatterns by Scanning Electrochemical Microscopy. Chemistry of Materials 13: 747-752.

${ }_{9}^{9}$ Wuu Y-M, Fan FRF, and Bard AJ (1989) High-resolution deposition of polyaniline on Pt with scanning electrochemical microscope. Journal of the Electrochemical Society 136: 885-886.

${ }^{10}$ Basame SB, and White HS (1999) Scanning Electrochemical Microscopy of Metal/Metal Oxide Electrodes. Analysis of Spatially Localized Electron-Transfer Reactions during Oxide Growth. Analytical Chemistry 71: 3166-3170.

${ }^{11}$ Gyurcsanyi RE, Jagerszki G, Kiss G, and Toth K (2004) Chemical imaging of biological systems with the scanning electrochemical microscope. Bioelectrochemistry 63: 207-215.

${ }^{12}$ Takii Y, Takoh K, Nishizawa M, and Matsue T (2003) Characterization of local respiratory activity of PC12 neuronal cell by scanning electrochemical microscopy. Electrochimica Acta 48: 3381-3385.

${ }^{13}$ Casillas N, Charlebois S, Smyrl W-H, and White H-S (1994) Pitting Corrosion of Titanium. Journal of the Electrochemical Society 141: 636-642.

${ }^{14}$ Fernandez JL, Imaduwage KP, and Zoski CG (2015) Carbon supported noble metal (Pd and Au) catalysts synthesized by an oxide route with high performance for oxygen reduction in acidic media. Electrochimica Acta 180: 460-470.

${ }^{15}$ Leonard KC, and Bard AJ (2013) The Study of Multireactional Electrochemical Interfaces via a Tip Generation/Substrate Collection Mode of Scanning Electrochemical Microscopy: The Hydrogen Evolution Reaction for Mn in Acidic Solution. Journal of the American Chemical Society 135: 15890 15896.

${ }^{16}$ Mirkin MV, and Wang Y (2012) Chapter 5: Theory. In Bard AJ, and Mirkin MV Editors. Scanning Electrochemical Microscopy. 2nd ed, pp 75-126. New York: Taylor and Francis.

${ }^{17}$ Lefrou C, and Cornut R (2010) Analytical Expressions for Quantitative Scanning Electrochemical Microscopy (SECM). Journal of chemical Physics and Physical Chemistry 11: 547-556.

${ }^{18}$ Minguzzi A, Alpuche-Aviles MA, Rodriguez-Lopez J, Rondinini S, and Bard AJ (2008) Screening of Oxygen Evolution Electrocatalysts by Scanning Electrochemical Microscopy Using a Shielded Tip Approach. Analytical Chemistry 80: 4055-4064.

${ }^{19}$ Sanchez CM, Rodriguez-Lopez J, and Bard AJ (2008) Scanning electrochemical microscopy. 60. quantitative calibration of the SECM substrate generation/tip collection mode and its use for the study of the oxygen reduction mechanism. Analytical Chemistry 80: 3254-3260. 
${ }^{20}$ Zhou F, Unwin PR, Bard AJ (1992) Scanning electrochemical microscopy. 16. Study of secondorder homogeneous chemical reactions via the feedback and generation/collection modes. Journal of Physical Chemistry 96: 4917-4924.

${ }^{21}$ Unwin PR (2012) Visualizing and quantifying homogeneous chemical reactions in electrochemical processes. In Bard AJ, and Mirkin MV Editors. Scanning Electrochemical Microscopy. 2nd ed, pp 157-190. New York: Taylor and Francis.

${ }^{22}$ Rodrigue-Lopez J, Zoski CG, and Bard AJ (2012) Application to electrocatalysis and photocatalysis and surface interrogation. In Bard AJ, and Mirkin MV Editors. Scanning Electrochemical Microscopy. 2nd ed, pp 525-568. New York: Taylor and Francis.

23 Fernandez JL, and Bard AJ (2003) Scanning Electrochemical Microscopy. 47. Imaging Electrocatalytic Activity for Oxygen Reduction in an Acidic Medium by the Tip Generation-Substrate Collection Mode. Analytical Chemistry 75: 2967-2974.

${ }^{24}$ Mirkin MV, Fan FRF, and Bard AJ ((1992) Direct Electrochemical Measurements inside a 2000Angstrom thick polymer film by scanning Electrochemical Microscopy. Science 257: 364-366.

${ }^{25}$ Lee CL, Kwak J, and Bard AJ (1990) Application of scanning electrochemical microscopy to biological samples. Proceedings of National Academy of Sciences of the United States of America 87: 1740-1743.

${ }^{26}$ Roberts WS, Lonsdale DJ, Griffiths J, and Higson SP (2007) Advances in the application of scanning electrochemical microscopy to bioanalytical systems. Biosensors \& Bioelectronics 23: 301318.

${ }^{27}$ Yasakawa T, Kaya T, Matsue T (2000) Characterization and Imaging of Single Cells with Scanning Electrochemical Microscopy. Electroanalysis 12: 653-659.

${ }^{28}$ Torisawa YS, Kaya T, Takii Y, Oyamatsu D, Nishizawa M, and Matsue T (2003) Scanning Electrochemical Microscopy-Based Drug Sensitivity Test for a Cell Culture Integrated in Silicon Microstructures. Analytical Chemistry 75: 2154-2158.

${ }^{29}$ Shiku H, Shiraishi T, Ohya H, Matsue T, Abe H, Hoshi H, and Kobayashi M (2001) Oxygen Consumption of Single Bovine Embryos Probed by Scanning Electrochemical Microscopy. Analytical Chemistry 73: 3751-3758.

${ }^{30}$ Mandler D (2001) Micro and Nanopatterning using the scanning electrochemical microscope. In Bard AJ, and Mirkin MV Editors. Scanning Electrochemical Microscopy. 1st ed, pp 593-627. New York: Marcel Dekker.

${ }^{31}$ Slim C, Tran Y, Chehimi M, Garraud N, Roger JP, Combellas C, and Kanoufi F (2008) Microelectrochemical Patterning of Surfaces with Polymer Brushes. Chemistry of Materials 20: 66776685.

${ }^{32}$ El-Giar EM, Said RA, Bridges GE, Thomson DJ (2000) Localized Electrochemical Deposition of Copper Microstructures. Journal of Electrochemical Society 147: 586-591.

33 Hüsser OE, Craston DH, and Bard AJ (1989) Scanning Electrochemical Microscopy: High-Resolution Deposition and Etching of Metals. Journal of Electrochemical Society 136: 32223229.

${ }^{34}$ Wuu YM, Fan FR, Bard AJ (1989) High resolution deposition of Polyaniline on Pt with the scanning electrochemical microscope. Journal of Electrochemical Society136: 885-886.

${ }^{35}$ Sugimura H, Uchida T, Shimo N, Kitamura N, and Masuhara H (1992) Fluorescent micropattern formation on ionic conductive polymer films by a scanning electrochemical microscope. Ultramicroscopy 468: 42-44

${ }^{36}$ Matrab T, Combellas C, and Kanoufi F (2008) Scanning electrochemical microscopy for the direct patterning of a gold surface with organic moities derived from iodonium salt. Electrochemistry Communications 10: 1230-1234.

37 Bard AJ, Fan FRF, Mirkin MV (Eds.) (1994) Scanning electrochemical Microscopy. In Electroanalytical Chemistry Vol.18. New York: Marcel Dekker.

${ }^{38}$ Schuhmann W, Kranz C, Wohlschlager H, Strohmeier J (1997) Pulse technique for the electrochemical deposition of polymer films on electrode surfaces. Biosensors \& Bioelectronics 12: $1157-1167$. 
39 Combellas C, Kanoufi F, Mazouzi D (2004) Surface Modification of Halogenated Polymers. 8. Local Reduction of Poly(tetrafluoroethylene) by the Scanning Electrochemical Microscope Transient Investigation. Journal of Physical Chemistry B 108: 19260-19268.

${ }^{40}$ Slim C, Ratajova E, Griveau S, Kanoufi F, Ferraro D, Perreard C, Varenne A, and Bedioui F (2015) Two-step local functionalization of fluoropolymer Dyneon THV microfluidic materials by scanning electrochemical microscopy combined to click reaction. Electrochemistry Communications 60: 5-8.

${ }^{41}$ Ebejer N, Schnippering M, Colburn AW, Edwards MA, Unwin PR (2010) Localized high resolution electrochemistry and multifunctional imaging: scanning electrochemical cell microscopy. Analytical Chemistry 82:9141-9145.

${ }^{42}$ Snowden ME, Güell AG, Lai SCS, McKelvey K, Ebejer N, O'Connell MA, Colburn AW, and Unwin PR (2012) Scanning electrochemical cell microscopy: theory and experiment for quantitative high resolution spatially-resolved voltammetry and simultaneous ion-conductance measurements.

Anaytical Chemistry 84, 2483-2491.

${ }^{43}$ Ebejer N, Güell AG, Lai SCS, McKelvey K, Snowden ME, and Unwin PR (2013) Scanning electrochemical cell microscopy: a versatile technique for nanoscale electrochemistry and functional imaging , 6. In Annual Review of Analytical Chemistry. Edited by Cooks RG, Pemberton JE: Annual Reviews, Palo Alto; 329-351.

${ }^{44}$ Kranz C (2014) Recent advancements in nanoelectrodes and nanopipettes used in combined scanning electrochemical microscopy techniques. Analyst 139: 336-352.

45 Bentley CL, Kang M, Unwin PR (2017) Scanning electrochemical cell microscopy: New perspectives on electrode processes in action. Current Opinion in Electrochemistry 6: 23-30.

${ }^{46}$ Kang M, Momotenko D, Page A, Perry D, and Unwin PR (2016) Frontiers in Nanoscale Electrochemical Imaging: Faster, Multifunctional, and Ultrasensitive. Langmuir 32: 7993-8008

${ }^{47}$ Güell AG, Meadows KE, Dudin PV, Ebejer N, Macpherson JV, and Unwin PR (2014) Mapping Nanoscale Electrochemistry of Individual Single-Walled Carbon Nanotubes. Nano Letters 14: 220224.

48 McKelvey K, O’Connell MA and Unwin PR (2013) Meniscus confined fabrication of multidimensional conducting polymer nanostructures with scanning electrochemical cell microscopy (SECCM). Chemical Communications 49: 2986-2988.

${ }^{49}$ Kirkman PM, Güell AG, Cuharuc AS, and Unwin PR (2014) Spatial and Temporal Control of the Diazonium Modification of $\mathrm{sp}^{2}$ Carbon Surfaces. Journal of American Chemical Society 136: 36-39. 